\title{
The influence of bile acids homeostasis by cryptotanshinone-containing herbs
}

Chengcheng Gao, Tianheng Ma, Liqun Pang, Rui Xie*

Department of Gastroenterology, Huai'an First People's Hospital, Nanjing Medical University, 6 Beijing Road West, Huai'an, Jiangsu 223300, P. R. China

\begin{abstract}
Background: Herbs might affect the homeostasis of bile acids through influence of multiple metabolic pathways of bile acids.

Objective: To investigate the inhibition of cryptotanshinone towards the glucuronidation of LCA, trying to indicate the possible influence of cryptotanshinone-containing herbs towards the homeostasis of bile acids.

Methods: The LCA-3-glucuronidation and LCA-24-glucuronidation reaction was monitored by LC-MS.

Results: Initial screening showed that $100 \mu \mathrm{M}$ of cryptotanshinone inhibited LCA-24-glucuronidation and LCA-3-glucuronidation reaction activity by $82.6 \%$ and $79.1 \%$, respectively. This kind of inhibition behaviour exerted cryptotanshinone concentrations-dependent and LCA concentrations-independent inhibition behaviour.

Conclusion: All these data indicated the possibility of cryptotanshinone's influence towards bile acids metabolism and homeostasis of bile acids.
\end{abstract}

Keywords: herbs, lithocholic acid (LCA), homeostasis

African Health Sciences 2014;14(1): 206-210 http://dx.doi.org/10.4314/ahs.v14i1.32

\section{Introduction}

Bile acids, initiated by CYP7A1-catalyzed cholesterol oxidation in liver, exert an important role in the solubilization, absorption, and transportation of dietary lipids in the intestine ${ }^{1}$. The most abundant bile acids in human are consisted of cholic acid (CA), chenodeoxycholic acid (CDCA), deoxycholic acid (DCA), and lithocholic acid (LCA) ${ }^{1}$. The homeostasis of bile acids can be tightly regulated through feed-back and feed-forward regulation pathways. Bile acids exert their toxicity towards cells at high concentrations, and the accumulation of bile acids can induce the severe damage towards liver cells ${ }^{2}$.

Bile acids have been reported to induce cell injury through many mechanisms, including the damage to the plasma membrane, oxidative stress, apoptosis, and inflammation ${ }^{3,4}$. Among the bile acids, LCA has been commonly considered as the most toxic bile acid which has widely used as the inducer of cholestasis ${ }^{5}$. Herbs can affect the metabolism of bile acids. For example, glycyrrhizin exhibited protective role towards LCA-induced liver injury through pregnane

\section{* Corresponding author:}

\section{Rui Xie}

Department of Gastroenterology, Huai'an First People's Hospital, Nanjing Medical

University, 6 Beijing Road West, Huai'an, Jiangsu

223300, P. R. China

E-mail: alpinetin139@163.com
$\mathrm{X}$ receptor (PXR) mediated-transcription regulation of cytochrome $3 \mathrm{~A}$ which has been regarded as the most important enzyme involved in the metabolism of LCA 6.

Besides CYP3A-catalyzed metabolism of LCA, glucuronidation of LCA has been regarded as another important metabolic pathway of LCA. The LCA-3-glucuronide (LCA-3G) and LCA-24-glucuronide (LCA$24 \mathrm{G})$ are the major glucuronides ${ }^{7}$. The inhibition of the glucuronidation of LCA might increase the toxicity induced by LCA. To date, no report was given on the influence of herbs on the glucuronidation of LCA. Danshen is a perennial plant in the genus Salvia, highly valued for its roots in traditional Chinese medicine. The present study aims to evaluate the inhibition of danshen's major ingredient cryptotanshinone as an example towards the glucuronidation of LCA.

\section{Materials and Methods \\ Reagents}

Lithocholic acid (LCA), uridine-5'-diphosphoglucuronic acid (UDPGA) (trisodium salt), Tris- $\mathrm{HCl}$, alamethicin from Trichoderma viride (purity $\geq 98 \%$ ) were purchased from Sigma-Aldrich (St Louis, MO). Pooled human liver microsomes (pHLMs) were purchased from BD Gentest. Cryptotanshinone was obtained from Shanghai Tauto Biotech Co. (Shanghai, China).

Evaluation of inbibitory effect of cryptotanshinone towards the LCA glucuronidation reaction

As previously described ${ }^{8}$, the incubation mixture (total volume $=100 \mu \mathrm{L})$ contained $50 \mathrm{mM}$ Tris- $\mathrm{HCl}(\mathrm{pH}=7.4)$, 
$10 \mathrm{mM} \mathrm{MgCl}, 25 \mu \mathrm{g}$ of alamethicin, $0.5 \mathrm{mg} / \mathrm{ml}$ HLMs, and LCA $(100 \mu \mathrm{M})$. All the glucuronidation incubations were performed under $37^{\circ} \mathrm{C}$ for $1 \mathrm{~h} .200 \mu \mathrm{L}$ ice-cold methanol was used to terminate the reaction. After centrifugation at $12000 \mathrm{rpm}$, the aliquots $(10 \mu \mathrm{L})$ of supernatant were used to undergo UPLC-MS analysis. For UPLC analysis, the Shim-pack XR-ODS II column $(75 * 2.0 \mathrm{~mm}, 2.2 \mu \mathrm{m})$ was used. The flow rate was set as $0.3 \mathrm{ml} / \mathrm{min}$. The eluents were methanol (A) and $0.2 \%$ formic acid (B) with the following gradients: 0-4 min, $35-70 \%$ A; 4-8 min, 70-80\% A; 8-12min, 80-95\% A; $12-$ $15 \mathrm{~min}, 35 \% \mathrm{~A}$. The negative mode was used to monitor LCA and its glucuronides. The ions [M-H] 375.1 and 551.1 were selected to monitor LCA and its glucuronides, respectively.

\section{Results}

Under recent analysis condition, LCA was eluted at 2.3 min, and its glucuronides LCA-3-glucuronide (LCA-3 -G) and LCA-24-glucuronide (LCA-24-G) were eluted at $1.15 \mathrm{~min}$ and $1.35 \mathrm{~min}$, respectively. $100 \mu \mathrm{M}$ of cryptotanshinone was firstly employed to investigate the inhibition towards LCA glucuronidation. $82.6 \%$ inhibition was detected for LCA-24-glucuronidation reaction (Fig. 1), and $79.1 \%$ activity of LCA-3-glucuronidation activity was inhibited (Fig. 3). Furthermore, the inhibition capability of various concentrations of cryptotanshinone towards the glucuronidation metabolism of LCA at different concentrations was investigated. Significant dose-dependent inhibition behaviour was observed for crytotanshinone (Fig. 2), however, the concentrationdependent inhibition behaviour for the LCA concentration was not observed (Fig. 4).

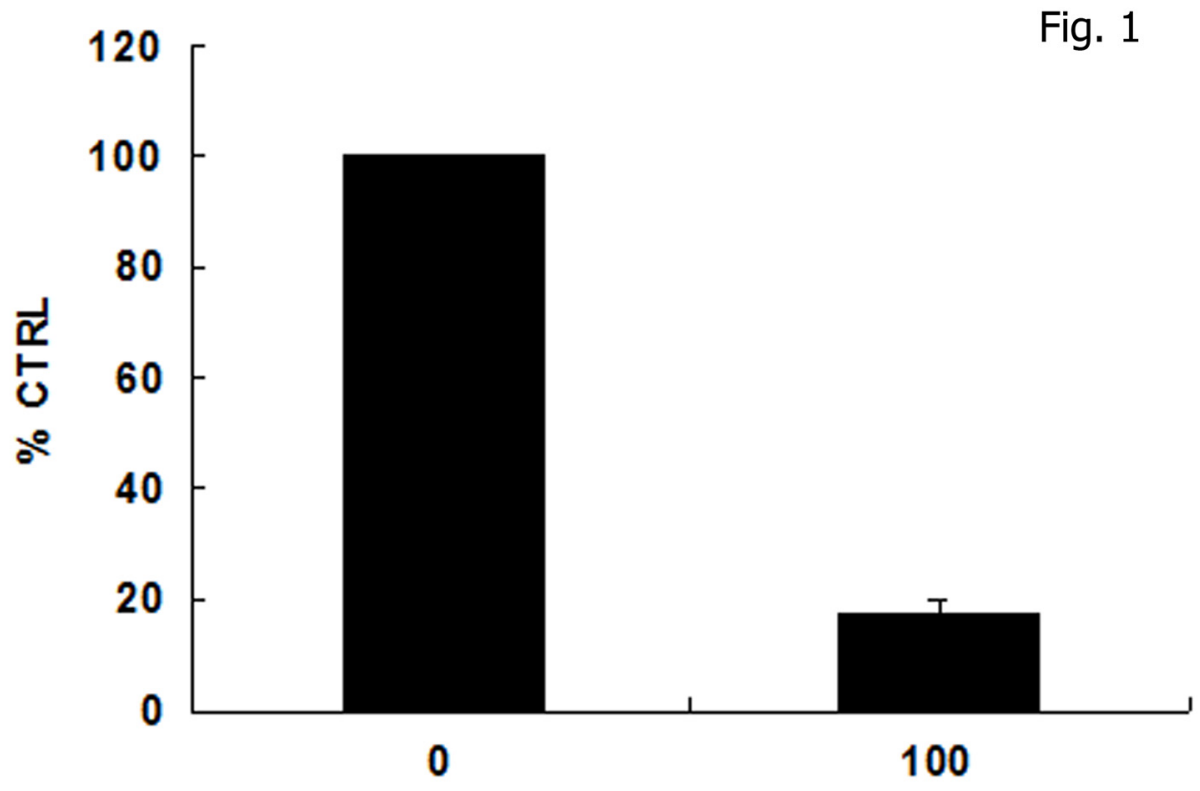

Fig. 1 The initial screening of cryptotanshinone's inhibitory capability towards the LCA-24-glucuronidation reaction activity. $50 \mu \mathrm{M}$ of LCA was used, and $100 \mu \mathrm{M}$ of cryptotanshinone was used. The data were given as mean plus S.D.. 


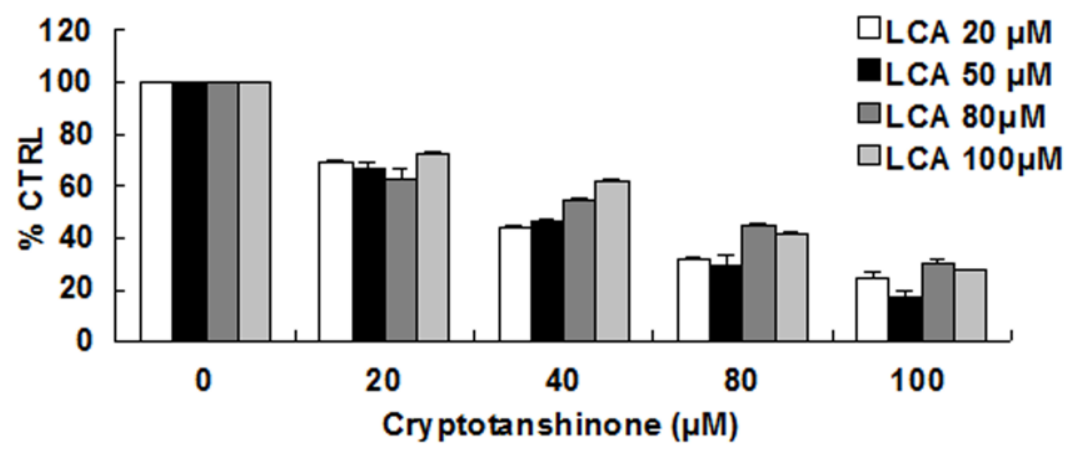

Fig. 2

Fig. 2 Different inhibitory potential of various concentrations of cryptotanshinone towards the LCA-24-glucuronidation reaction activity. Various concentrations of cryptotanshinone $(0,20,40,80$ and $100 \mu \mathrm{M})$ and LCA (20, 50, 50, and $100 \mu \mathrm{M})$ were used.

Fig. 3

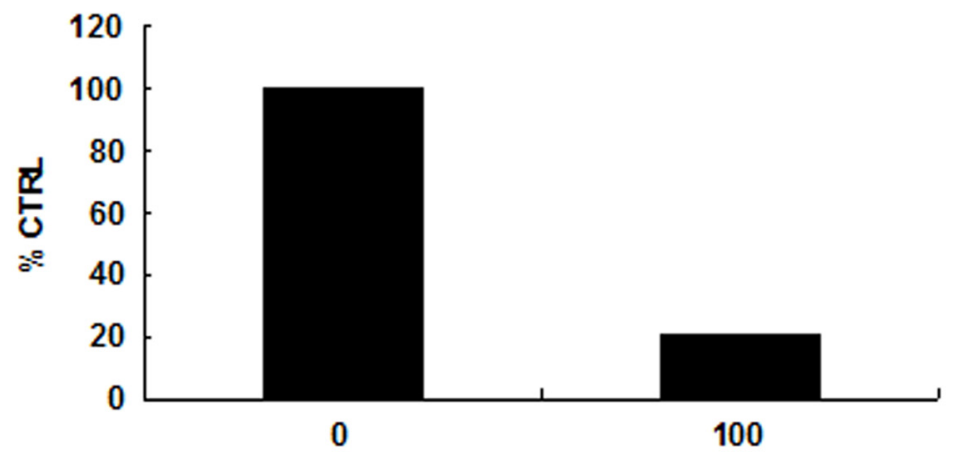

Fig. 3 The initial screening of cryptotanshinone's inhibitory capability towards the LCA-3-glucuronidation reaction activity. $50 \mu \mathrm{M}$ of LCA was used, and $100 \mu \mathrm{M}$ of cryptotanshinone was used. The data were given as mean plus S.D.. 


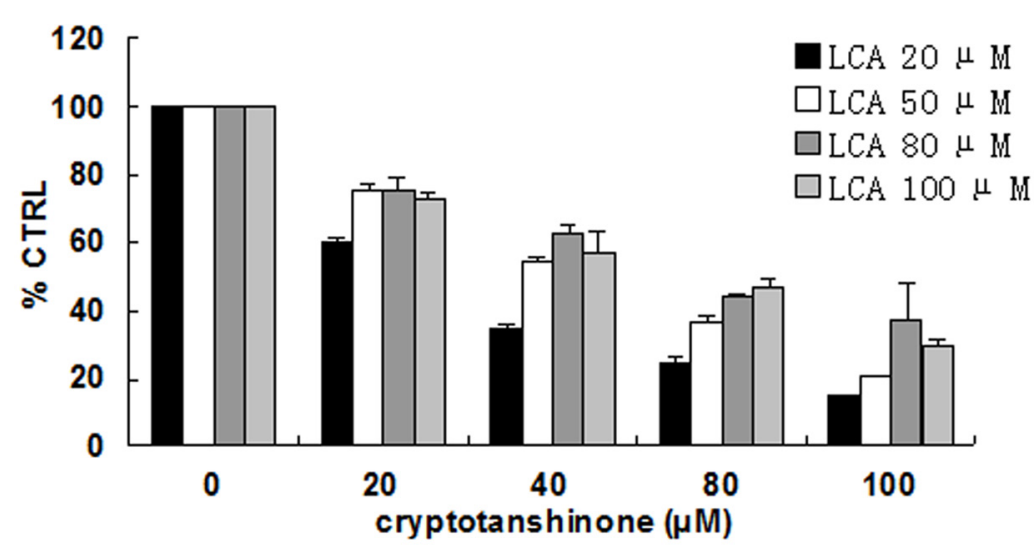

Fig. 4

Fig. 4 Different inhibitory potential of various concentrations of cryptotanshinone towards the LCA-3glucuronidation reaction activity. Various concentrations of cryptotanshinone $(0,20,40,80$ and $100 \mu \mathrm{M})$ and LCA $(20,50,50$, and $100 \mu \mathrm{M})$ were used.

\section{Discussion}

Multiple metabolic pathways catalyzed by enzymes contributed to the homeostasis of bile acids. The xenobiotics can affect the serum level of bile acids through influence of all these pathways, including synthesis and detoxification pathways. For example, herbs can up-regulate the activity of CYP7A1 which accelerate the synthesis of bile acids 9. Glycyrrhizin, a major ingredient of licorice, can increase the detoxification process of LCA through accelerating CYP3A-catalyzed metabolism of $\mathrm{LCA}^{6}$.

The present study focused on the glucuronidation detoxification of bile acids, using LCA as a typical substrate. LCA can undergo efficient glucuronidation process in 3-OH and 24-OH. Therefore, the inhibition potential of crytotanshinone towards both LCA-3glucuronidation and LCA-24-glucuronidation reactions was investigated in the present study. Strong inhibition of crytotanshinone towards LCA-3-glucuronidation and LCA-24-glucuronidation was demonstrated, and this kind of inhibition was demonstrated to be crytotanshinone concentrations-dependent and LCA concentrations-independent. This result showed the possible disturbance of crytotanshinone towards the metabolism of LCA, indicating the possible influence of crytotanshinone towards the homeostasis of bile acids. It should be noted that the enzymes involved in the metabolism of LCA (e.g. UGT1A3) were also involved in the metabolism of other bile acids (e.g., chenodeoxycholic acid) ${ }^{10}$. Therefore, danshen might also disturb the homeostasis of bile acids through inhibition of other bile acids' metabolism.

It should be noted that the present study results should be explained in the context of herbal complexity. Many other components in herbs might complicate the final influence of herbs towards the homeostasis of bile acids. Additionally, the long administration of herbs might also up-regulate the enzymes involved in the glucuronidation of LCA through important nuclear receptors (e.g., farnesoid X receptor (FXR), peroxisome proliferator-activated receptor alpha (PPARalpha), etc.). For example, nuclear receptor farnesoid X receptor (FXR) has been reported to activate the activity of UGT1A3 which is a key enzyme involved in the glucuronidation of LCA ${ }^{10}$.

\section{References}

Russell DW. The enzymes, regulation, and genetics of bile acid synthesis. Annu Rev Biochem 2003; 72: $137-$ 174.

2. Trottier J, Caron P, Straka RJ, Barbier O. Profile of serum bile acids in noncholestatic volunteers: genderrelated differences in response to fenofibrate. Clin Pharmacol Ther 2011; 90(2):279-286.

3. Allen K, Jaeschke H, Copple BL. Bile acids induce inflammatory genes in hepatocytes: a novel mechanism 
of inflammation during obstructive cholestasis. Am J Pathol 2011; 178: 175-186.

4. Perez MJ, Briz O. Bile-acid-induced cell injury and protection. World J Gastroenterol 2009; 15: 1677 1689.

5. Matsubara T, Tanaka N, Sato M, Kang DW, Krausz KW, Flanders KC, Ikeda K, Luecke H, Wakefield LM, Gonzalez FJ. TGF- $\beta$-SMAD3 signaling mediates hepatic bile acid and phospholipid metabolism following lithocholic acid-induced liver injury. J Lipid Res 2012; 53(12):2698-2707.

6. Wang YG, Zhou JM, Ma ZC, Li H, Liang QD, Tan HL, Xiao CR, Zhang BL, Gao Y. Pregnane X receptor mediated-transcription regulation of CYP3A by glycyrrhizin: a possible mechanism for its hepatoprotective property against lithocholic acid-induced injury. Chem Biol Interact 2012; 200(1):11-20.

7. Perreault M, Gauthier-Landry L, Trottier J, Verreault
M, Caron P, Finel M, Barbier O. The Human UDPGlucuronosyltransferase UGT2A1 and UGT2A2 Enzymes Are Highly Active in Bile Acid Glucuronidation. Drug Metab Dispos 2013; 41(9):1616-1620.

8. Caron P, Trottier J, Verreault M, Bélanger J, Kaeding J, Barbier O. Enzymatic production of bile Acid glucuronides used as analytical standards for liquid chromatography-mass spectrometry analyses. Mol Pharm 2006; 3(3):293-302.

9. Guo J, Bei W, Hu Y, Tang C, He W, Liu X, Huang L, Cao Y, Hu X, Zhong X, Cao L. A new TCM formula FTZ lowers serum cholesterol by regulating HMGCoA reductase and CYP7A1 in hyperlipidemic rats. J Ethnopharmacol 2011; 135(2):299-307.

10. Erichsen TJ, Aehlen A, Ehmer U, Kalthoff S, Manns MP, Strassburg CP. Regulation of the human bile acid UDP-glucuronosyltransferase $1 \mathrm{~A} 3$ by the farnesoid X receptor and bile acids. J Hepatol 2010;52(4):570-578. 